Provided for non-commercial research and education use. Not for reproduction, distribution or commercial use.

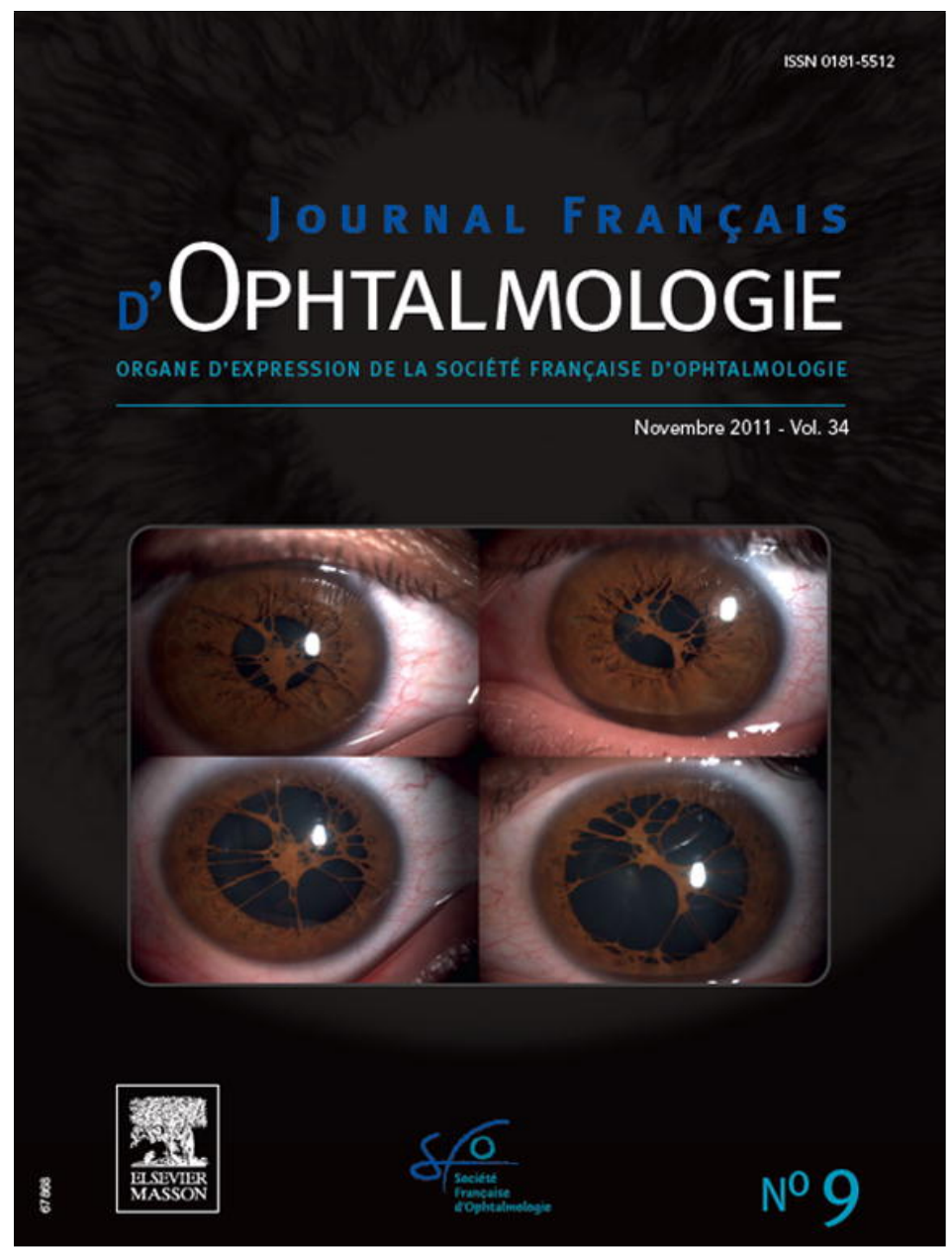

This article appeared in a journal published by Elsevier. The attached copy is furnished to the author for internal non-commercial research and education use, including for instruction at the authors institution and sharing with colleagues.

Other uses, including reproduction and distribution, or selling or licensing copies, or posting to personal, institutional or third party websites are prohibited.

In most cases authors are permitted to post their version of the article (e.g. in Word or Tex form) to their personal website or institutional repository. Authors requiring further information regarding Elsevier's archiving and manuscript policies are encouraged to visit:

http://www.elsevier.com/copyright 
Disponible en ligne sur

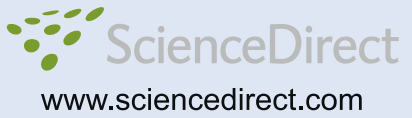

Elsevier Masson France

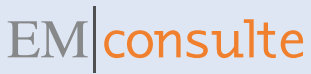

www.em-consulte.com

COMMUNICATION DE LA SFO

\title{
Adénocarcinome de la glande lacrymale dans un rétinoblastome bilatéral traité avec radiothérapie externe: à propos d'un cas ${ }^{\text {is }}$
}

\section{Adenocarcinoma of the lacrimal gland in bilateral retinoblastoma treated with external radiotherapy: A case report}

\author{
D.M. Bubella*, R.M. Bubella, S. Carità, F. Di Giovanna, \\ L. Di Rosa, G. Lodato
}

Dipartimento di Neuroscienze Cliniche (DUNC), Sezione di Oftalmologia, Università degli Studi di Palermo, Via Liborio Giuffrè 13, 90127 Palermo, Italy

Reçu le 9 avril 2009 ; accepté le 14 juin 2011

Disponible sur Internet le 5 octobre 2011

\section{MOTS CLÉS}

Adénocarcinome de la glande lacrymale ;

Radiothérapie ;

Rétinoblastome

bilatéral

\section{KEYWORDS}

Adenocarcinoma of the lacrimal gland;

Radiotherapy;

Bilateral

retinoblastoma
Résumé Dans ce cas clinique, nous voulons décrire les caractéristiques cliniques et les particularités diagnostiques d'un adénocarcinome de la glande lacrymale développé 20 ans après une radiothérapie externe pour le traitement d'un rétinoblastome bilatéral. L'acuité visuelle, la biomicroscopie à la lampe à fente, la photographie en couleur du fond d'œil, la résonance magnétique, l'orbitotomie latérale et l'analyse histologique de la tumeur sont présentées. (c) 2011 Elsevier Masson SAS. Tous droits réservés.
Summary This clinical case report describes the clinical findings and diagnosis of lacrimal gland adenocarcinoma that developed 20 years after external beam radiotherapy in the treatment of bilateral retinoblastoma. Visual acuity, slit lamp biomicroscopy, fundus color photography, nuclear magnetic resonance, lateral orbitotomy and histological analysis are described.

(c) 2011 Elsevier Masson SAS. All rights reserved.

\footnotetext{
Communication orale présentée lors du $114^{\mathrm{e}}$ congrès de la Société française d'ophtalmologie en mai 2008.

* Auteur correspondant.

Adresse e-mail : morreale.bubella@libero.it (D.M. Bubella).
} 


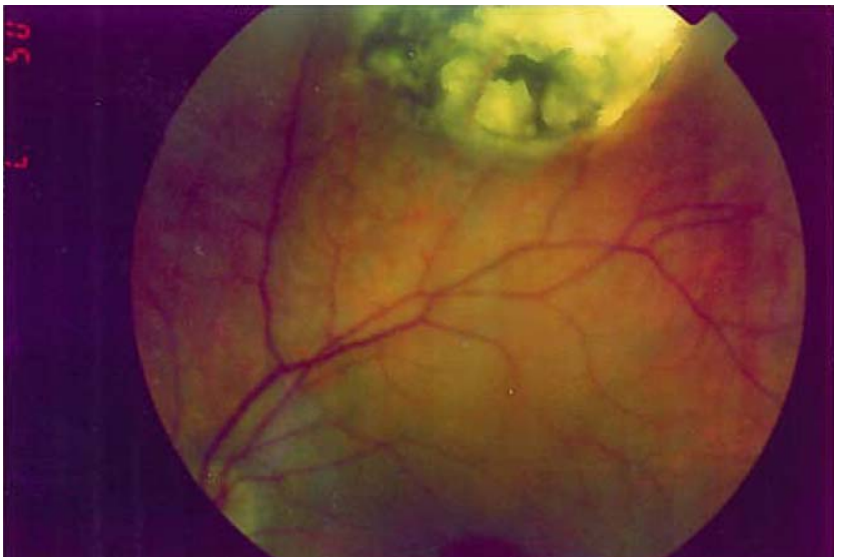

Figure 1. Photographie en couleur du fond d'oeil, OG: zone d'atrophie choriorétinienne secondaire à une photocoagulation au laser argon avec radiothérapie externe.

\section{Introduction}

L'adénocarcinome de la glande lacrymale est une tumeur rare qui représente environ $5-7 \%$ des tumeurs épitheliales de la glande lacrymale $[1,2]$. On expose le cas d'un adénocarcinome de la glande lacrymale chez une jeune femme avec ayant un antecédent de rétinoblastome bilatéral traité par énucléation, photocoagulation au laser, chimiothérapie et radiothérapie externe dans l'enfance.

\section{Cas clinique}

Nous avons observé une femme de 23 ans aux antécédents de rétinoblastome bilatéral dans la deuxième année de vie, traitée par énucléation de l'œil droit et traitement conservateur par photocoagulation au laser argon avec radiothérapie (3000 rad) de l'œil gauche et chimiothérapie systémique (carboplatin et étoposide). En mai 2007, elle présentait une tuméfaction en regard de la paupière supérieure gauche depuis six mois, avec un aspect en $\mathrm{S}$, non douloureuse et sans exophtalmie.

L'acuité visuelle était de 0,4 LogMar non améliorable. Il n'y avait pas de limitation de l'oculomotricité. L'examen à la lampe à fente montrait un segment antérieur normal avec un réflexe photomoteur présent. Au fond d'œil, on remarquait la présence d'une cicatrice (zone d'atrophie choriorétinienne) secondaire à une photocoagulation au laser argon pour le traitement d'un rétinoblastome (Fig. 1). L'imagerie par résonance magnétique retrouvait, en coupe axiale et coronale, un processus tumoral orbitaire situé en regard de la glande lacrymale gauche avec un aspect hypo-intense en $\mathrm{T} 1$ et hyper-intense en $\mathrm{T} 2$, avec prise de contraste intensive après l'administration du gadolinium (Fig. 2). La masse tumorale n'envahissait pas les parois osseuses.

Une intervention chirurgicale a été réalisée sous anesthésie générale. L'abord chirurgical été antérieur, par le pli palpébral supérieur, étendu à une ostéotomie de Kronlein et a permis l'exérèse complète de la glande lacrymale. Les suites opératoires ont été simples. Après un recul de 12 mois, on ne notait aucune récidive. L'examen anatomopathologique

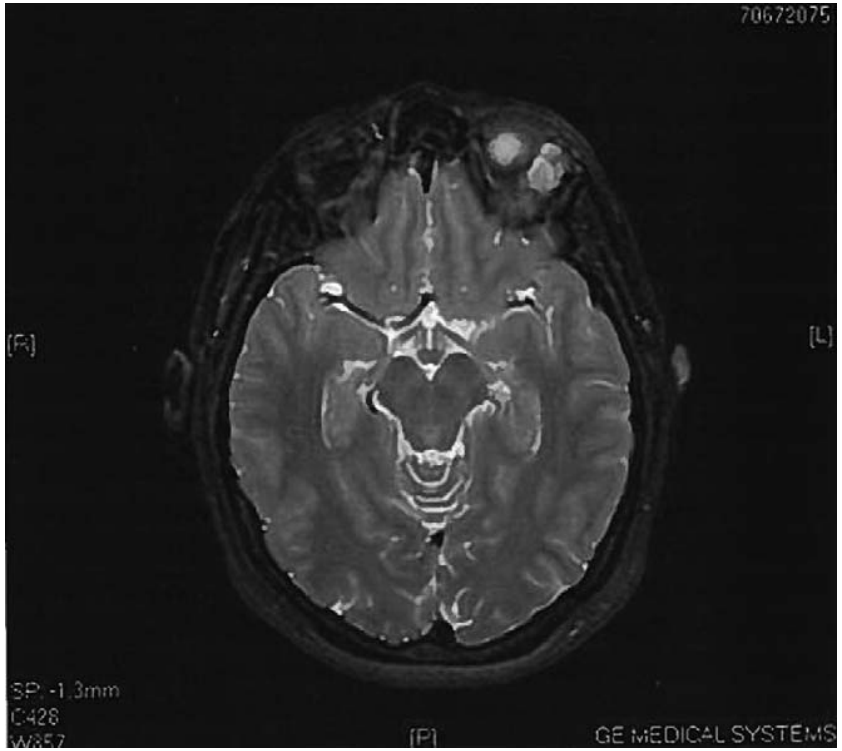

Figure 2. Résonance magnétique orbitaire (coupe axiale). Processus tumoral orbitaire situé en regard de la glande lacrymale gauche. Les parois osseuses ne sont pas intéressées par la masse tumorale. Prothèse oculaire dans la cavité orbitaire droite.

montrait la présence d'un adénocarcinome au sein d'un adénome pléomorphe de la glande lacrymale (Fig. 3A et B).

L'examen immunohistochimique a permis le diagnostic d'adénocarcinome, avec notamment :

- protéine-S100: positif ;

- alpha actin: focal positive ;

- pancytokeratin: positive.

Un bilan carcinologique exhaustif était entrepris à la recherche de métastases. Le scanner corps entier et la scintigraphie osseuse ne montraient aucune localisation secondaire.

\section{Discussion}

Le rétinoblastome est la plus fréquente des tumeurs malignes intraoculaires de l'enfant. Les enfants avec une mutation du gene $r b$ ont un risque accru de développer pendant la vie, des sarcomes des tissus mous et des os, des mélanomes et des tumeurs cérébrales avec un risque augmenté respectivement de 300 fois, de 100 fois et de 24 fois par rapport à la population générale [3].

Howrey et al. [4] estiment l'incidence d'une seconde néoplasie à $51 \%$ à 50 ans du diagnostic du rétinoblastome. Kleinerman et al. [5] révèlent que l'incidence cumulative de développer une tumeur secondaire à 50 années du diagnostic du rétinoblastome est de $36 \%$ pour les formes héréditaires et de $5,7 \%$ pour les formes non héréditaires. En plus, la radiotherapie augmenterait le risque d'une tumeur secondaire de 3,1 fois dans les formes héréditaires. Aussi, Marees et al. [6] révèlent que la radiothérapie induit un risque de seconde néoplasie (hazard ratio [HR] $=2,81$ ).

Dans les observations de Imhof et al. [7], seuls les rétinoblastomes héréditaires traités avec radiothérapie externe développent une seconde tumeur. Imhof et al. [8], de plus, 

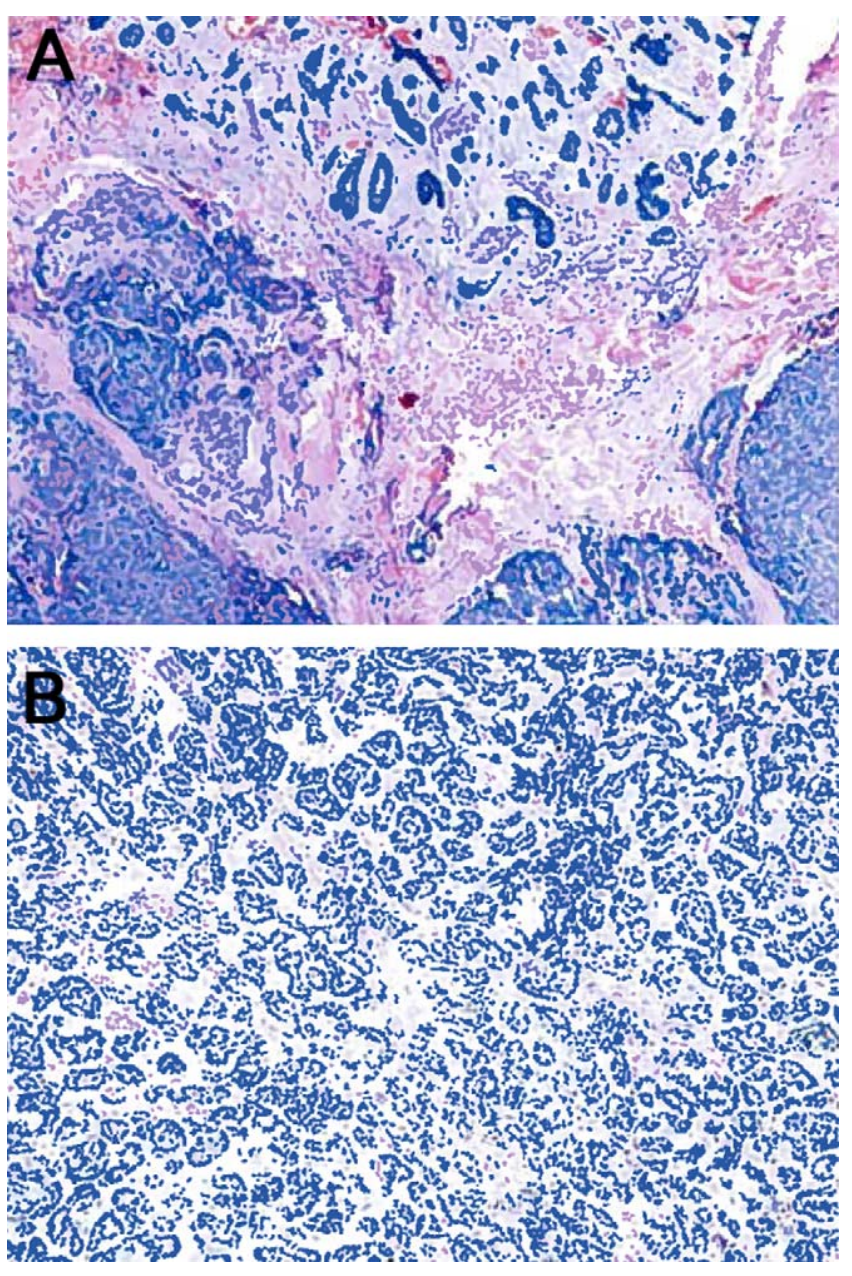

Figure 3. Examen histologique après coloration par hématoxyline-éosine. A. Présence de tubes glandulaires isolés au sein d'un stroma myxoïde, translucide, rose avec occasionnelles cellules myoépithéliales en fusées et infiltration carcinomateuse $(\times 10)$. B. Néoplasie carcinomateuse peu différentiée avec aspect de pénétration et destruction au sein de l'adénome pléomorphe contigu $(\times 40)$.

ont montré que l'irradiation externe modifie la glande lacrymale en réduisant sa production des larmes.

Aussi, Mohney et al. [9] décrivent la croissance d'une seconde néoplasie chez les sujets avec un rétinoblastome héréditaire et pensent que la faible incidence relevée, dans leur série, pourrait être liée à la technique utilisée, et au dosage de la radiothérapie.

Moll et al. [10] remarquent que la radiothérapie précoce, avant 12 mois d'âge, augmente le risque de tumeurs secondaires ( 22 versus $3 \%$ ), cependant, ils notent que ce risque serait lié davantage au champ d'irradiation qu'à l'âge.

Aertes et al. [11], récemment, ont relevé que la radiothérapie devrait être utilisée exclusivement pour des tumeurs étendues compte tenu des risques que cette thérapie implique. Dans notre cas, l'examen anatomopathologique et l'examen immuno-histochimique ont éliminé une métastase de rétinoblastome. La formation d'un adénocarcinome au sein d'un adénome pleomorphe de la glande lacrymale pourrait être le résultat des mutations chromosomiques radio-induites sur des anomalies géniques héréditaires.
On peut présumer, donc, que l'adénome pléomorphe de la glande lacrymale, déterminé par la radiothérapie, ait été la première lésion tumorale liée à la radiothérapie. Notre observation est proche de celle de Hadjistilianou et al. [12] qui ont décrit la croissance d'un adénome pléomorphe 17 ans après la radiothérapie pour le traitement d'un rétinoblastome bilatéral chez une jeune femme.

Galatoire et al. [13], en effet, ont décrit un cas d'adénocarcinome au sein d'un adénome pléomorphe de la glande lacrymale et affirment que même si cette éventualité est rare, il faut la considérer.

Selon Rootman [14], la transformation maligne intervient dans moins de $3 \%$ des cas sur dix ans et dans 10 à $20 \%$ des cas sur 20 ans.

\section{Conclusion}

Le risque d'apparition d'une seconde tumeur non oculaire liée à la radiothérapie justifie la recherche dans le traitement du rétinoblastome d'autres voies thérapeutiques, comme la chimiothérapie sélective intra-artérielle, la chimioréduction, la thermochimiothérapie [15] et la chimiothérapie combinée à la cyclosporine bien que parmi les drogues utilisées, à l'heure actuelle, certaines sont mutagènes et pourraient provoquer des cancers secondaires [16].

Il est préférable d'utiliser dans l'enfance, pour l'étude diagnostique, l'IRM et l'échographie, en évitant les scanners pour ne pas irradier inutilement ces enfants.

\section{Déclaration d'intérêts}

Les auteurs n'ont pas transmis de déclaration de conflits d'intérêts.

\section{Références}

[1] Lee V, Hungerford JL, Bunce C, Ahmed F, Kingston JE, Plowman PN. Globe conserving treatment of the only eye in bilateral retinoblastoma. Br J Ophtalmol 2003;11:1374-80.

[2] Amaddeo P, Fontana S. Orbital tumors: review of the literature and presentation of our experience. Minerva Oftalmol 2009;51:59-78.

[3] Eng C, Li FP, Abramson D. Mortality from second tumours among long-term survivors of retinoblastoma. J Natl Cancer Inst 1993;85:1121-8.

[4] Howrey RP, Lipham WJ, Schultz WH, Buckley EG, Dutton JJ, Klintworth GK, et al. Sebaceous gland carcinoma: a subtle second malignancy following radiation therapy in patients with bilateral retinoblastoma. Cancer 1998;83:767-71.

[5] Kleinerman RA, Tucker MA, Tarone RE, Abramson DH, Seddon JM, Stovall M, Li FP, Fraumeni Jr JF. Risk of new cancers after radiotherapy in long-term survivors of retinoblastoma: an extended follow-up. J Clin Oncol 2005;23:2272-9.

[6] Marees T, Moll AC, Imhof SM, de Boer MR, Ringens PJ, van Leeuwen FE. Risk of second malignancies in survivors of retinoblastoma: more than 40 years of follow-up. J Natl Cancer Inst 2008;100:1771-9.

[7] Imhof SM, Moll AC, Hofman P, Mourits MP, Schipper J, Tan KE. Second primary tumours in hereditary - and non hereditary retinoblastoma patients treated with megavoltage external beam irradiation. Doc Ophthalmol 1997;93:337-44. 
[8] Imhof SM, Hofman P, Tan KE. Quantification of lacrimal function after $\mathrm{D}$ shaped gfield irradiation for retinoblastoma. $\mathrm{Br} \mathrm{J}$ Ophthalmol 1993;77:482-4.

[9] Mohney BG, Robertson DM, Schomberg PJ, Hodge DO. Second monocular tumors in survivors of heritable retinoblastoma prior radiation therapy. Am J Ophthalmol 1998;126:230-7.

[10] Moll AC, Imhof SM, Schouten-Van Meeteren AY, Kuik DJ, Hofman $P$, Boers $M$. Second primary tumors in hereditary retinoblastoma: a register-based study, 1945-1997: is there an effect on radiation related risk? Ophthalmology 2001;108:1109-14.

[11] Aertes I, Lumbroso L, Le Rouic L, Gautier-Villars M, Brisse H, Doz F, Desiardins L. Retinoblastoma. Orphanet J Rare Dis 2006;1:31-8.

[12] Hadjistilianu T, de Francesco S, Signori C, Menicacci F, Galluzzi P, Toti P. Pleomorphic adenoma of the lacrimal gland in an 18-year-old girl irradiated for bilateral retinoblastoma. Orbit 2006;25:51-3.

[13] Galatoire O, Hamédani M, Putterman M, Berges O, Morax $S$. Adénocarcinome au sein d'un adénome pléomorphe de la glande lacrymale. À propos d'un cas. J Fr Ophtalmol 2005;28:896-901.

[14] Rootman J. Lacrimal gland adenocarcinoma. Disease of the orbit, second edition, 2003. p. 351-60.

[15] Lumbroso L, Doz F, Levi C, Dendale R, Vedrenne J, Bours $\mathrm{D}$, et al. Thermothérapie et thermochimiotherapie au laser diode dans le traitement du retinoblastoma. J Fr Ophtalmol 2003;26:154-9.

[16] Wong FL, Boice JD, Abramson DH, Tarone RE, Kleinerman RA, Stovall $M$, et al. Cancer incidence after retinoblastoma: radiation dose and sarcoma risk. JAMA 1997;278: 1262-7. 